\title{
Séquences varvaires du Lac Barlow et Moraine de Laverlochère : déglaciation tardive de la partie nord du lac Témiscamingue, Québec Varved Sequences of Lake Barlow and the Laverlochère Moraine: Deglaciation of the Northern Area of Lake Témiscamingue, Québec \\ Warwen-Sequenz des Barlow-Sees und der Laverlochère-Moräne: späte Enteisung des nördlichen Teils des Témiscamingue-Sees, Québec
}

\section{Robert-André Daigneault et Gilbert Prichonnet}

\section{Volume 42, numéro 2, 1988}

URI : https://id.erudit.org/iderudit/032718ar

DOI : https://doi.org/10.7202/032718ar

\section{Aller au sommaire du numéro}

\section{Éditeur(s)}

Les Presses de l'Université de Montréal

ISSN

0705-7199 (imprimé)

1492-143X (numérique)

\section{Découvrir la revue}

\section{Citer cet article}

Daigneault, R.-A. \& Prichonnet, G. (1988). Séquences varvaires du Lac Barlow et Moraine de Laverlochère : déglaciation tardive de la partie nord du lac Témiscamingue, Québec. Géographie physique et Quaternaire, 42(2), 107-120. https://doi.org/10.7202/032718ar

\section{Résumé de l'article}

La déglaciation du secteur nord du lac Témiscamingue est précisée par une étude sédimentologique. Deux séquences varvaires, situées à PE du lac et distantes de $10 \mathrm{~km}$ sont corrélées grâce aux variations de l'épaisseur des lits d'été et de la teneur en carbonate du dépôt qui est commandée par la quantité de silt : 5,5 fois plus de silt et 3,7 fois plus de carbonate dans les lits d'été que dans les lits d'hiver. Néanmoins, la fraction carbonatée augmente dans le temps sans variation significative du pourcentage de silt. La présence tardive d'un lobe glaciaire centré sur le lambeau de roches paléozoïques du fossé tectonique du Témiscamingue s'harmoniserait donc avec la disposition spatiale des deux séquences varvaires et expliquerait l'augmentation de la teneur en carbonate. La corrélation probable de ces séquences varvaires avec celles des séries Temiscaming B4 et C4 d'Antevs confirmerait la présence tardive de glace dans le secteur. La dispersion des galets de roches paléozoïques dans les segments de la Moraine de Laverlochère permet, par ailleurs, de démontrer un faible transport glaciaire vers PE (secteur N) et vers le SE (secteur SE), confirmé par la bimodalité des teneurs en carbonate dans les fractions plus fines des accumulations granulaires. Cependant, dans les accumulations situées à PE, on note une dispersion des paléocourants, la présence exclusive de galets précambriens et un faible pourcentage de débris carbonates dans les fractions fines. Les segments orientaux et subméridiens de la Moraine de Laverlochère s'apparentent donc tout autant aux trains d'eskers régionaux qu'à une limite d'arc morainique. Irrégularité de la marge glaciaire et ralentissement du retrait sont deux phénomènes caractéristiques du recul de l'inlandsis dans la région, à l'Holocène inférieur. 


\section{SÉQUENCES VARVAIRES DU LAC BARLOW ET MORAINE DE LAVERLOCHËRE: DÉGLACIATION TARDIVE DE LA PARTIE NORD DU LAC TÉMISCAMINGUE, QUÉBEC}

Robert-André DAIGNEAULT et Gilbert PRICHONNET, Département des sciences de la Terre, Université du Québec à Montréal, C.P. 8888, Succursale «A», Montréal, Québec H3C 3P8.

RÉSUMÉ La déglaciation du secteur nord du lac Témiscamingue est précisée par une étude sédimentologique. Deux séquences varvaires, situées à l'E du lac et distantes de $10 \mathrm{~km}$ sont corrélées grâce aux variations de l'épaisseur des lits d'été et de la teneur en carbonate du dépôt qui est commandée par la quantité de silt: 5,5 fois plus de silt et 3,7 fois plus de carbonate dans les lits d'été que dans les lits d'hiver. Néanmoins, la fraction carbonatée augmente dans le temps sans variation significative du pourcentage de silt. La présence tardive d'un lobe glaciaire centré sur le lambeau de roches paléozoïques du fossé tectonique du Témiscamingue s'harmoniserait donc avec la disposition spatiale des deux séquences varvaires et expliquerait l'augmentation de la teneur en carbonate. La corrélation probable de ces séquences varvaires avec celles des séries Temiscaming B4 et C4 d'Antevs confirmerait la présence tardive de glace dans le secteur. La dispersion des galets de roches paléozoïques dans les segments de la Moraine de Laverlochère permet, par ailleurs, de démontrer un faible transport glaciaire vers I'E (secteur $N$ ) et vers le SE (secteur SE), confirmé par la bimodalité des teneurs en carbonate dans les fractions plus fines des accumulations granulaires. Cependant, dans les accumulations situées à l'E, on note une dispersion des paléocourants, la présence exclusive de galets précambriens et un faible pourcentage de débris carbonatés dans les fractions fines. Les segments orientaux et subméridiens de la Moraine de Laverlochère s'apparentent donc tout autant aux trains d'eskers régionaux qu'à une limite d'arc morainique. Irrégularité de la marge glaciaire et ralentissement du retrait sont deux phénomènes caractéristiques du recul de l'inlandsis dans la région, à l'Holocène inférieur.
ABSTRACT Varved sequences of Lake Barlow and the Laverlochère Moraine: deglaciation of the northern area of Lake Témiscamingue, Québec. Sedimentological studies show that two varved sequences, located $10 \mathrm{~km}$ apart on the east side of the lake, are correlated on the basis of the change of summer layer thicknesses and the total carbonate content which is controlled by the amount of silt: the summer layers contain 5.5 times more silt than winter layers and are 3.7 richer in carbonate. Nevertheless, the carbonate content increases upward in the sequences of varves without a significant variation of the silty fraction. The increasing carbonate content is explained by the presence of a persistent ice lobe centred on the Paleozoic rocks of the Temiscamingue graben. The possible correlation of these varved sequences with the Temiscaming B4 and C4 series of Antevs might corroborate the presence of a persistent ice lobe in this region. The dispersal pattern of the Paleozoic pebbles of the ice-contact deposits of the Laverlochère Moraine also clearly demonstrate the short distance of transport by the ice: towards the $E$ (northern part) and the SE (southeastern part). This transport is also marked by bimodal distribution of the carbonate content in the finer fractions of these accumulations. However, in the eastern accumulations, we can observe a more random distribution of the paleocurrents, the absence of pebbles other than those of Precambrian origin and a low percentage of the carbonate content in the finer debris. The N-S orientation of the eastern segment could be associated either to the regional pattern of the eskers or to an arcuate morainic landform. Irregularity of the ice-front and lower rates of the ice retreat characterize the model of deglaciation in that region during the early Holocene.
ZUSAMMENFASSUNG Warwen-Sequenz des Barlow-Sees und der LaverlochèreMoräne: späte Enteisung des nördlichen Teils des Témiscamingue-Sees, Québec. Die Enteisung des nördlichen Teils des Témiscamingue-Sees wird duch eine Sediment-Studie verdeutlicht. Zwei Warwen-Sequenzen, die im Osten des Sees liegen und voneinander $10 \mathrm{~km}$ entfernt sind, werden zueinander in Wechselbeziehung gesetzt, dank der Variationen der Dicke der Sommer-Betten und dem Karbonat-Gehalt der Ablagerung, welcher durch den Schlamm-Gehalt bestimmt wird: $5,5 \mathrm{mal}$ mehr Schlamm und $3,7 \mathrm{mal}$ mehr Karbonat in den Sommer-Betten als in den Winter-Betten. Dennoch nimmt der Karbonat-Anteil allmählich zu, ohne dass sich der Schlamm-Anteil signifikant ändert. Das späte Vorkommen eines Eis-Lappens, der sich genau um die paläozoische Felsscholle des tektonischen Témiscamingue-Grabens konzentriert, würde also mit der räumlichen Verteilung der zwei Warwen-Sequenzen übereinstimmen und die Zunahme des Karbonat-Gehalts erklären. Die wahrscheinliche Korrelation dieser Warwen-Sequenzen mit denen der Témiscamingue C4-Serie von Antevs würde das späte Vorkommen von Eis in diesem Gebiet bestätigen. Die Zerstreuung der paläozoischen Fels-Gerölle in den Segmenten der Moräne von Laverlochère erlaubt ausserdem, einen geringen Eis-Transport nach Osten (nördlicher Teil) und nach Südosten (südöstlicher Teil) nachzuweisen, was durch die Bimodalität der Karbonat-Gehalte in den feineren Splittern der körnigen Ansammlungen bestätigt wird. Jedoch bemerkt man in den im Osten gelegenen Ansammlungen eine Zerstreuung der PaläoStrömungen, das ausschliessliche Vorkommen von präkambrischen Geröllen und einen geringen Anteil von karboniertem Geröll in den feineren Splittern. Die östlichen und submeridianen Segmente der LaverlochèreMoräne können also sowohl mit den regionalen Esker-Strömen als auch einer Grenze des Moränen-Bogens in Verbindung gebracht werden. 


\section{INTRODUCTION}

Au cours du dernier retrait glaciaire, une grande partie du Témiscamingue et de l'Abitibi fut recouverte par les lacs Barlow et Ojibway (Wilson, 1918; Prest, 1970; Vincent et Hardy, 1977): 2027 varves s'y sont accumulées (Antevs, 1925). Plusieurs corps fluvioglaciaires marquent également les étapes du retrait glaciaire (Veillette, 1983a et b): selon cet auteur, la Moraine de Laverlochère délimiterait la bordure est d'un lobe de glace tardif dans l'auge du lac Témiscamingue.

Cette étude présente des données relatives à deux séquences varvaires et à la Moraine de Laverlochère. Les résultats de ces analyses permettent de discuter l'hypothèse de la présence du lobe de glace tardif et l'importance du transport qu'il aurait entraîné.

\section{CADRE PHYSIQUE}

La zone d'étude se situe au Témiscamingue, dans le nordouest québécois; elle est comprise entre $47^{\circ} 15^{\prime} \mathrm{N}$ et $47^{\circ} 40^{\prime} \mathrm{N}$ et entre $79^{\circ} 10^{\prime} \mathrm{O}$ et $79^{\circ} 45^{\prime} \mathrm{O}$ (fig. $1 \mathrm{~A}$ et 2). Du point de vue physiographique, elle couvre une partie de la plaine de Cobalt (Bostock, 1972). Celle-ci, d'une altitude variant de 180 à 305 m, est bordée à l'E comme à l'O par un relief de plateaux montueux, les hautes terres laurentidiennes, dont l'altitude moyenne

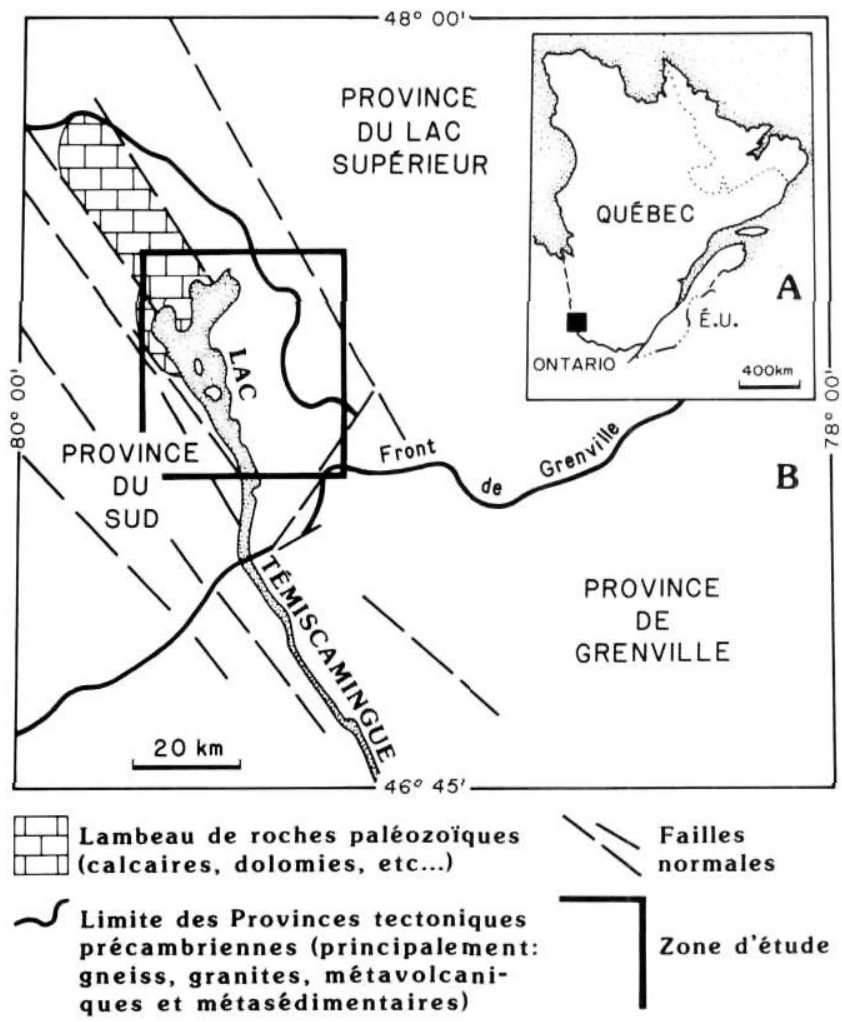

FIGURE 1. A) Localisation de la zone d'étude; B) Aperçu de la géologie du substratum rocheux (modifiée à partir de Lovell et Caine, 1970; Mc Glynn, 1972; Donaldson et Munro, 1972).

A) Location of the study area. B) Geology of the bedrock (modified from Lovell and Caine, 1970; Mc Glynn, 1972; Donaldson and Munro, 1972). est d'environ $335 \mathrm{~m}$ (fig. 3). Cette topographie s'explique par la présence de longues failles parallèles d'orientation NO-SE qui délimitent un fossé d'effondrement centré sur le lac Témiscamingue (fig. 1B). Ce graben est associé à ceux d'OttawaBonnechère et de la vallée du Saint-Laurent (Kay, 1942; Kumarapelli et Saull, 1966): il a permis la préservation d'un lambeau de roches paléozoïques au NO du lac Témiscamingue: les roches carbonatées de cette série ont été utilisées comme traceurs dans l'étude des mouvements glaciaires.

Les autres lithologies régionales appartiennent aux provinces tectoniques du Sud ou du Lac Supérieur (fig. 1B): ce sont respectivement des roches sédimentaires d'âge protérozoïque (Supergroupe de l'Huronien; McGlynn, 1972) et des roches de type granitoïde ou métavolcanique (Avrantchev et Lebel-Drolet, 1981). Les débris de roches protérozoïques ont également été utilisées comme traceurs lithologiques dans les dépôts fluvioglaciaires.

\section{TRAVAUX ANTÉRIEURS}

Les varves de la zone étudiée forment une épaisse couverture (max. $40 \mathrm{~m}$ environ), relativement continue, dans la vallée du lac Témiscamingue. Vers le nord, jusqu'au delà de l'actuelle ligne de partage des eaux entre les bassins versants

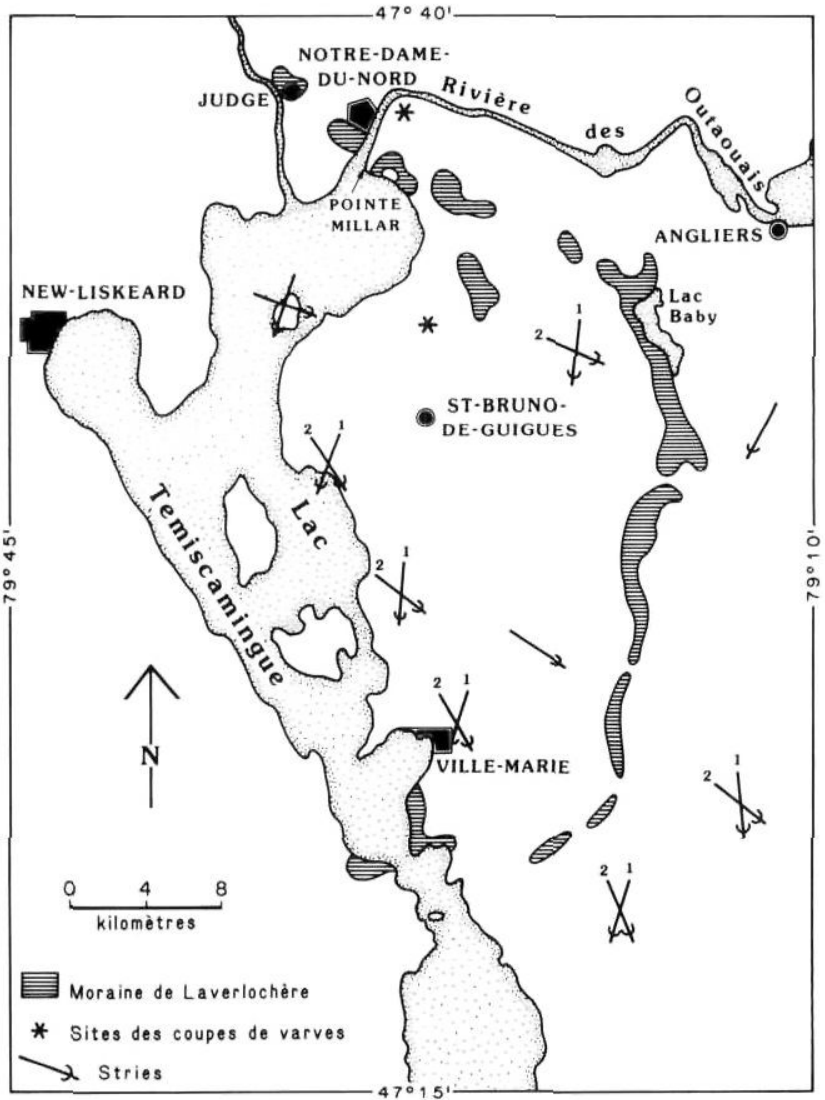

FIGURE 2. Quelques données de la géologie du Quaternaire (modifiée à partir de Veillette 1983a et 1986a). Localisation des coupes de varves échantillonnées.

Data of the Quaternary geology (modified from Veillette 1983a and 1986a). Location of the studied varve sections. 
de la baie de James et de la rivière des Outaouais, les varves se limitent à de petits placages isolés.

Coleman (1909) considérait que ces sédiments argileux s'étaient déposés dans une extension vers le NE du Lac Algonquin. La déglaciation des vallées des rivières des Outaouais et Mattawa aurait par la suite permis l'existence d'un lac de plus basse altitude, situé au nord de l'actuelle ligne de partage des eaux, qu'il appela Lac Ojibway.

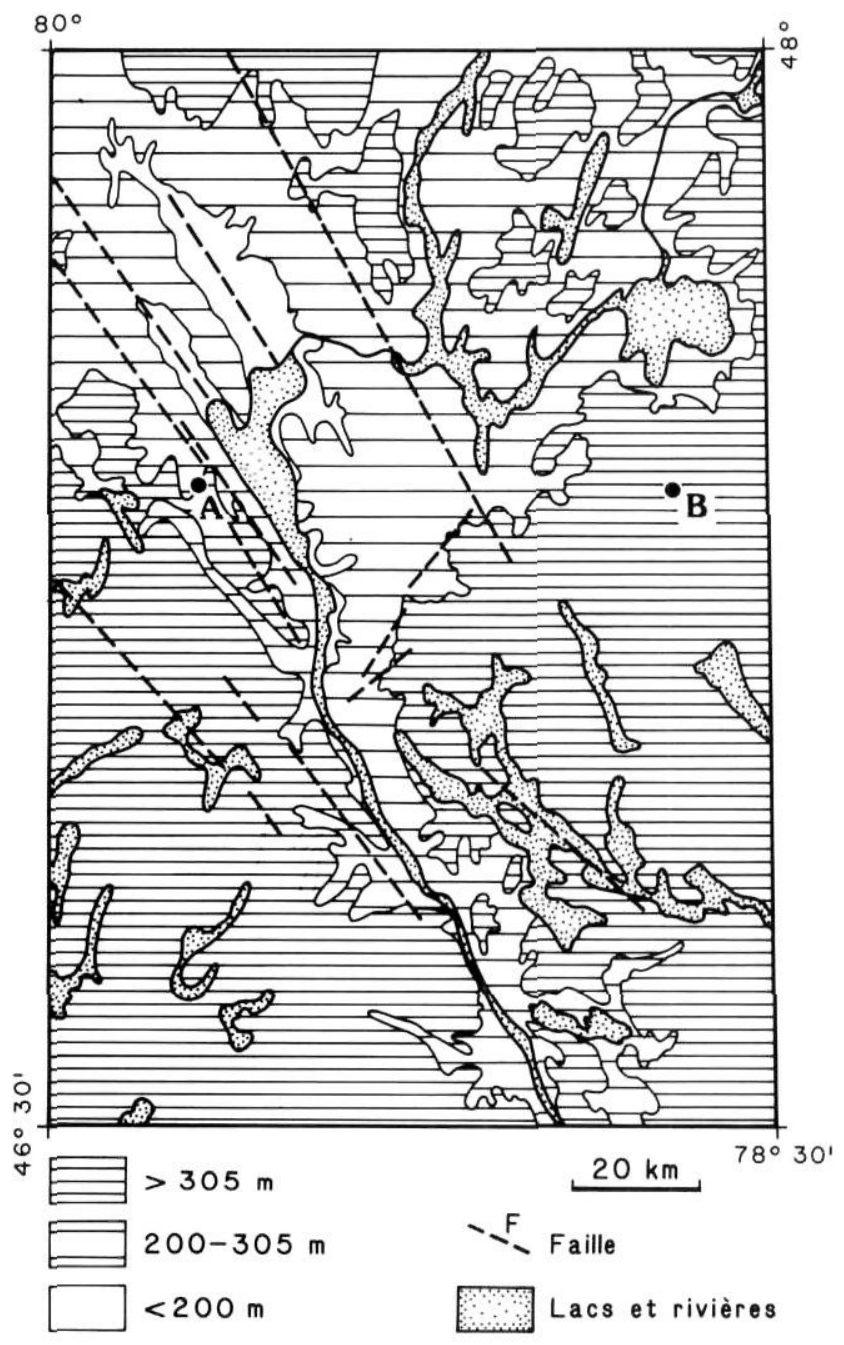

\section{A}

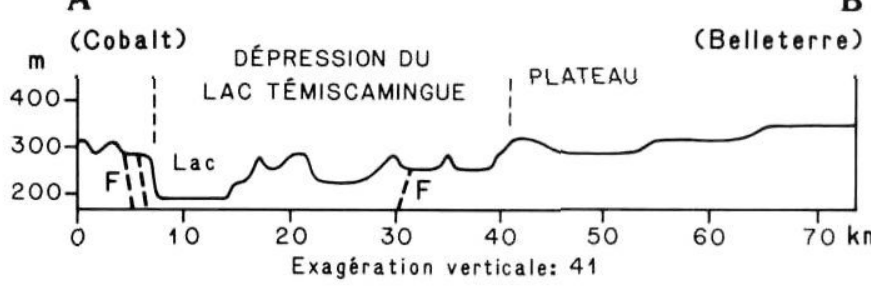

FIGURE 3. Physiographie du plateau précambrien et de la dépression du lac Témiscamingue: les coupes de varves sont situees dans la plaine basse $(<200 \mathrm{~m})$.

Physiography of the Precambrian plateau and Témiscamingue through: the sections of the varves are located in the lower plain $(<200 \mathrm{~m})$.
Wilson (1918) a montré que les sédiments glaciolacustres, au sud et au nord de la ligne de partage des eaux, appartiennent à un même grand bassin qu'il nomme le Lac Barlow; selon cet auteur ce dernier fut séparé du Lac Algonquin pendant la plus grande partie de son existence.

Antevs (1925) fut le premier à étudier de façon détaillée ces sédiments glaciolacustres: en se fondant sur l'épaisseur totale des varves observées en divers sites, il a établi des corrélations stratigraphiques et proposé une chronologie des événements tardiglaciaires de la région. II utilisait ainsi la méthode chronostratigraphique employée en Suède par De Geer dès 1884 (Lundqvist, 1985). Antevs supposa que le retrait de la marge glaciaire s'était fait à un taux annuel moyen de 138 m. Puis, en 1953, cet auteur, estima à 2550 ans la durée totale de l'épisode glaciolacustre du Lac Barlow.

Hughes (1965) proposa de nommer Formation de BarlowOjibway l'ensemble des sédiments glaciolacustres du Témiscamingue et de l'Abitibi. II reconnut qu'il y avait périodicité annuelle des varves en se fondant sur les travaux de Terasmae (1963) dans la région de Kirkland Lake: Terasmae avait démontré, en effet, une plus grande abondance des spores et du pollen dans les couches de silt que dans les couches d'argile. Reprenant les données d'Antevs (1925), Hughes (1965) proposa la date de moins 9350 ans pour le drainage du Lac "Barlow-Ojibway». Prest (1970), se fondant sur la disposition générale des niveaux glaciolacustres du centre du Canada, a déduit que l'épisode du Lac Barlow aurait débuté vers moins 10900 à 10600 ans et qu'il se serait étendu audelà de l'actuelle ligne de partage des eaux durant la phase qu'il nomme Barlow-Ojibway. Enfin, pour Vincent et Hardy (1977), c'est l'émersion du seuil d'Angliers qui scinda en deux les eaux du Lac Barlow; ce seuil commanda la première phase du Lac Ojibway et confina le Lac Barlow à l'auge du Témiscamingue.

Banerjee (1973) s'est principalement intéressé aux textures et aux structures des varves du "Lac Barlow-Ojibway". Selon lui, les courants de turbidité dans les lits d'été, et la suspension dans ceux d'hiver, sont bien les causes de la mise en place des sédiments.

La Moraine de Laverlochère est le nom donné par Veillette (1982, 1983 a et b) à une série d'accumulations de sédiments de contact glaciaire, disposées en arc de cercle, à l'est du lac Témiscamingue (fig. 2). L'auteur s'appuie principalement sur l'analyse des microformes d'érosion glaciaire pour associer l'ensemble de ces accumulations à une moraine plutôt qu'à un esker, comme l'avait précédemment proposé Vincent (1971). En effet, dans ce secteur, on note de nombreuses stries O-E et NO-SE recoupant les stries orientées NE-SO (considéré comme le mouvement régional lors du dernier épisode glaciaire; fig. 2). Ces directions d'écoulement plus récentes ne se retrouvent qu'en petit nombre immédiatement à l'est de la Moraine de Laverlochère. Elles semblent donc s'expliquer par la présence d'un lobe de glace à "écoulement radial» (Veillette, 1983b, p. 73), dont la limite serait marquée par cette moraine. Un retrait tardif de la glace dans ce secteur avait déjà été proposé par Barlow, en 1899. 
Selon Veillette (1982, 1983 a et b) un mode de retrait glaciaire caractérisé par une marge très irrégulière aurait affecté l'ensemble de la vallée supérieure de l'Outaouais. Rappelons que Boissonneau (1968) a proposé l'hypothèse d'un vaste rentrant dans la marge glaciaire, entre Sudbury et Mattawa, qui aurait permis l'extension d'un niveau lacustre post-Algonquin dans la vallée inférieure du lac Témiscamingue. Le modèle de Veillette s'oppose à celui d'Antevs (1925) et de Vincent et Hardy (1977): pour ces trois auteurs, une marge glaciaire "rectiligne" aurait reculé perpendiculairement à l'axe de la vallée.

\section{SÉQUENCES VARVAIRES ÉTUDIÉES}

\section{MÉTHODOLOGIE}

Les deux séquences varvaires étudiées (fig. 2) se situent respectivement à Saint-Bruno-de-Guigues (SBG; (fig. 4) et à Notre-Dame-du-Nord (NDN; fig. 5). Une distance de $10 \mathrm{~km}$ les sépare.

Afin de travailler dans des conditions optimales d'observation, les varves furent échantillonnées à l'aide de gouttières métalliques et analysées en laboratoire. Dans un premier temps, la paroi des coupes a été taillée verticalement à l'aide d'une truelle (fig. 5). Les boîtes furent ensuite enfoncées en évitant de déformer la gouttière métallique et les sédiments.

Pour obtenir un contraste maximal entre les couplets, les échantillons ont été séchés quelques heures en laboratoire (fig. 6) comme le recommande Hughes (1955). Afin de caractériser les varves, les observations et les analyses sédimentologiques suivantes ont été effectuées: mesure de l'épaisseur, structures internes, granulométrie, teneur totale en carbonate et minéralogie des argiles.

La granulométrie moyenne des varves (échantillon composé de trois varves successives) fut déterminée par densimétrie (méthode de Mériaux, 1954). La teneur totale en carbonate

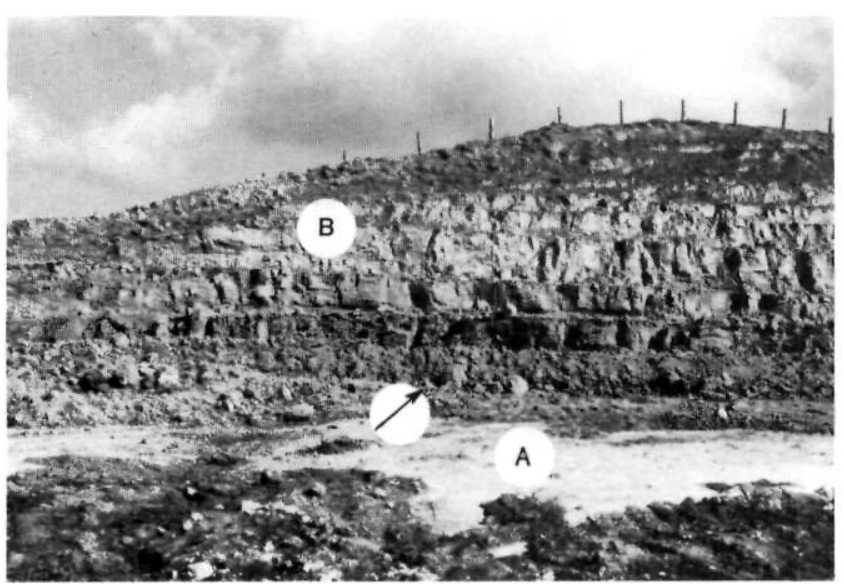

FIGURE 4. Coupe de Saint-Bruno-de-Guigues. A) substratum rocheux (calcaire dolomitique); B) séquence quaternaire (voir fig. 7). La flèche indique la localisation des premiers échantillons.

St-Bruno-de-Guigues section. A) Bedrock (dolomitic limestone); B) Quaternary sequence (see Fig. 7). The arrow indicates the location of the first sample. a été obtenue par attaque à l'acide dans un calcimètre de type Bernard, et la minéralogie des argiles par diffraction aux rayons $\mathrm{X}$.

\section{STRATIGRAPHIE DES COUPES}

La coupe de SBG surmonte un front de carrière du Paléozoïque (fig. 7): sa hauteur totale est de $12 \mathrm{~m}$. De la base (180 $\mathrm{m}$ asl) au sommet, on observe: le substratum rocheux (calcaire dolomitique); un till carbonaté de couleur gris-bleu (0-0,3 m); un diamicton glacio-lacustre $(0,3-0,5 \mathrm{~m})$; une séquence de varves proximales silteuses $(0,5-1,0 \mathrm{~m})$ contenant quelques galets de délestage; et enfin des varves distales $(1,0-12 \mathrm{~m})$. Ces dernières succèdent aux varves proximales sans zone de transition. Les six mètres supérieurs de cette coupe sont desséchés et fragmentés: les varves n'ont pu être échantillonnées. Environ $1 \mathrm{~m}$ de sable recouvre la séquence: en surface le sable forme une série de cordons littoraux. Le contact des sables sur les varves est probablement discordant.

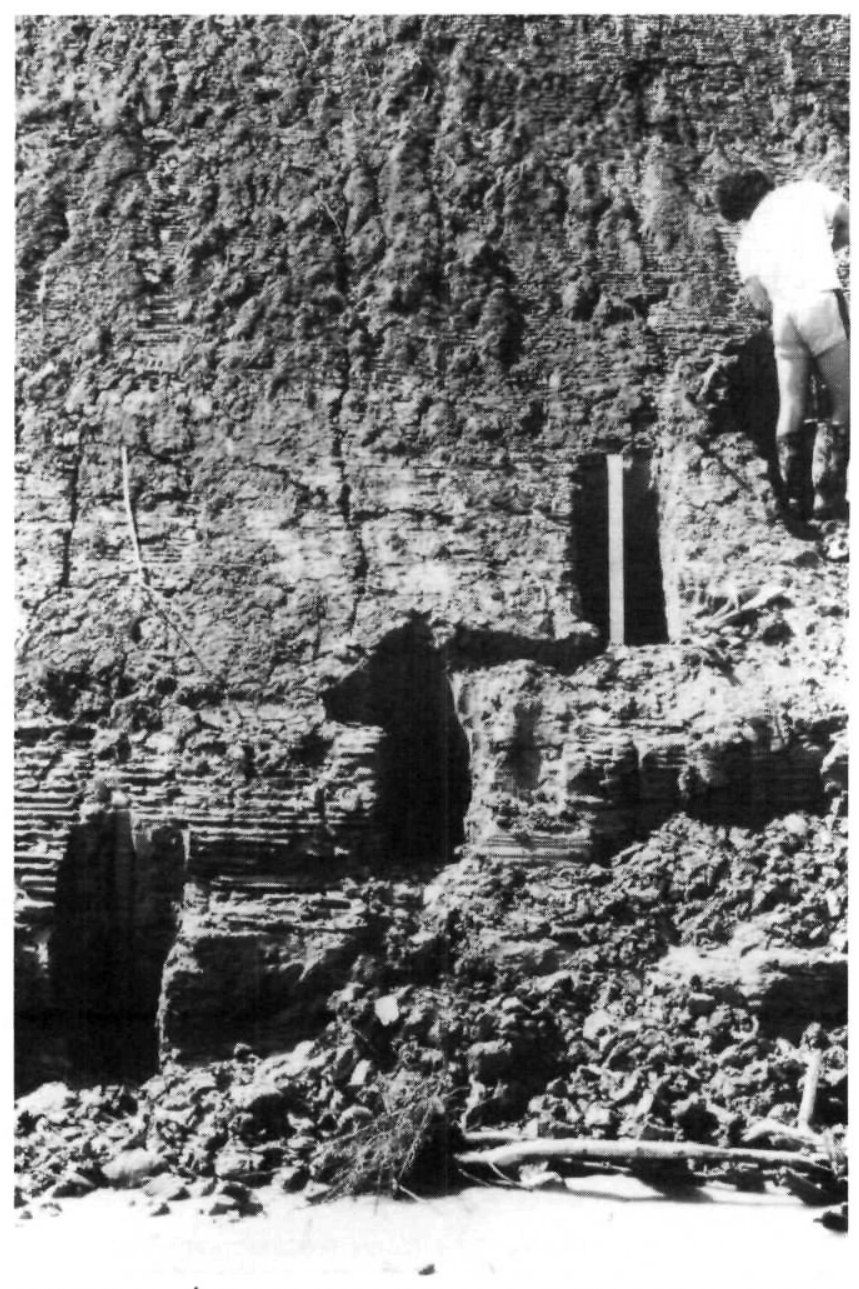

FIGURE 5. Échantillonnage de la coupe de Notre-Dame-du-Nord, à l'aide de gouttières métalliques: noter la régularité des varves.

Method of varve sampling with metal gutters at Notre-Dame-duNord section: note the regularity of the varves. 


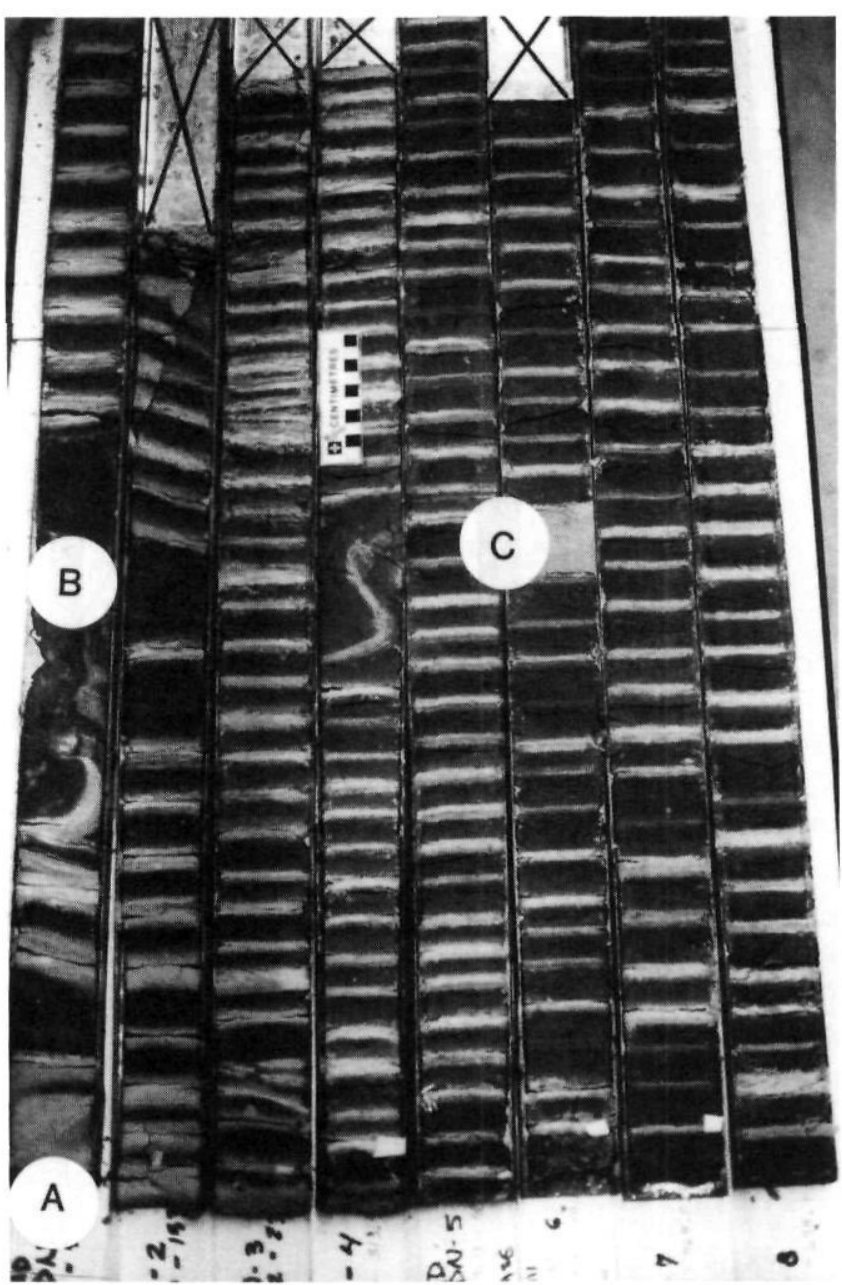

FIGURE 6. Début de la séquence varvaire à Notre-Dame-du-Nord. De gauche à droite. A) varves épaisses de la base; B) déformations; C) varve épaisse responsable du pic noté sur la courbe moyenne de la figure 11 (cf. varve 663);

Basal sequence of the varves at Notre-Dame-du-Nord (from left to right). A) Thicker varves at the base; B) deformation; C) thick varve (cf. varve 663) of the mean curve of Figure 11.

La coupe de NDN a une hauteur de $20 \mathrm{~m}$. À partir du niveau de l'eau de la rivière des Outaouais $(178,3 \mathrm{~m}$ asl) jusqu'à $19 \mathrm{~m}$, on observe des varves distales. Ces varves sont tronquées par des sables littoraux $(\approx 1 \mathrm{~m})$. Les dix mètres supérieurs ont été perturbés par un glissement de terrain: seuls les $10 \mathrm{~m}$ de la base ont donc été échantillonnés.

D'après notre interprétation des données de forages réalisées par Nenniger et Chênevert (1960), il semble que la séquence de varves distales au site de NDN débute à environ $1,5 \mathrm{~m}$ sous le niveau de l'eau.

\section{RÉSULTATS}

\section{Comptage}

Au site de SBG, environ 174 varves distales ont été comptées. En deux endroits, en raison de déformations, il a

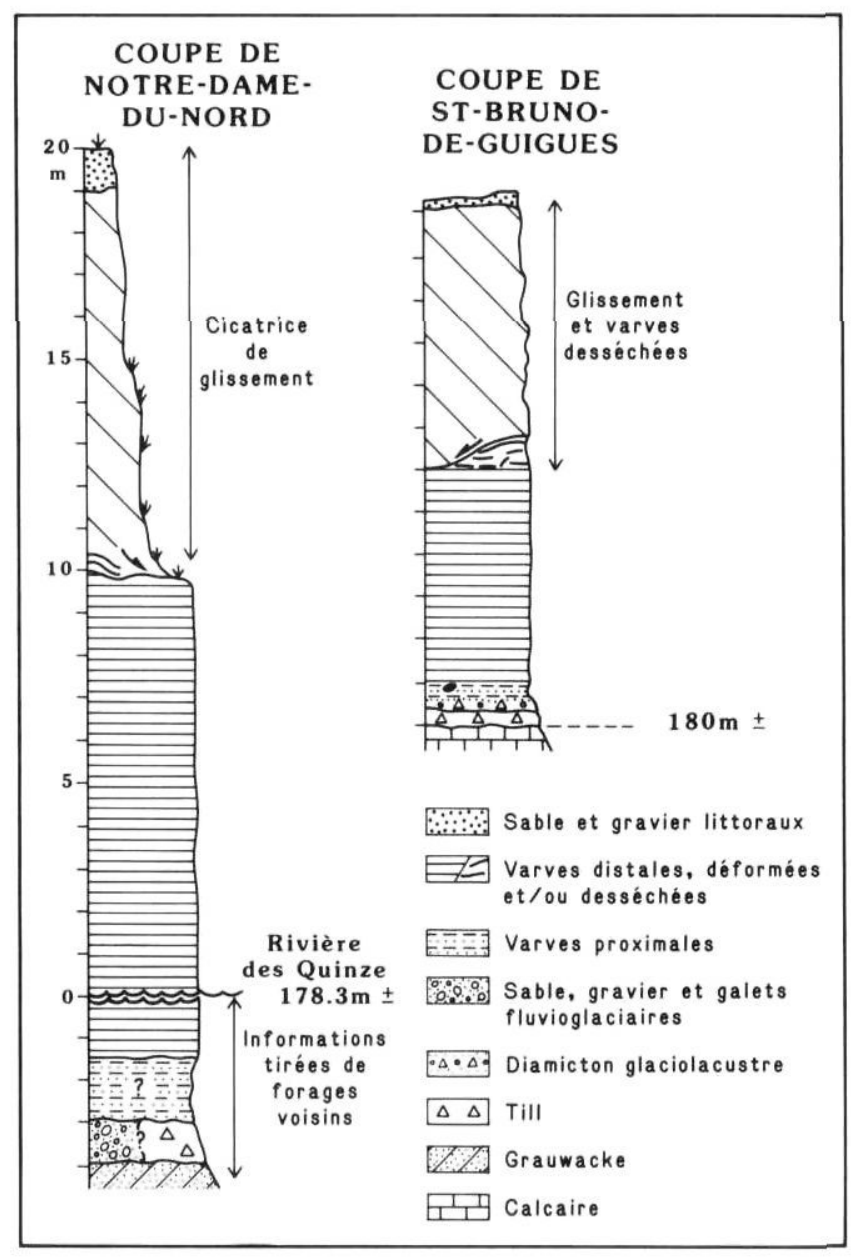

FIGURE 7. Coupes lithologiques des séquences étudiées.

Lithological sections of the studied sequences.

fallu procéder par approximation pour établir le nombre de varves impliquées: pour cela, l'épaisseur de sédiment perturbé a été mesurée et divisée par l'épaisseur moyenne des varves de part et d'autre de l'horizon déformé (5 varves au-dessous et 5 varves au-dessus). Dans les deux cas, environ 6 varves ont été déformées ou disloquées lors de glissements. À NDN 320 varves ont été mesurées et 13 évaluées selon la même méthode (trois déformations ou glissements sous-aquatiques?).

\section{Structures internes}

Les structures internes des varves sont identiques dans les deux sites: les lits d'été sont massifs mais intensément bioturbés; ils passent de façon graduelle aux lits d'hiver. La gradation se fait par l'alternance de microlaminations parallèles de silt et d'argile $(\approx 1 \mathrm{~mm})$. Les lits d'hiver sont également massifs mais sans bioturbations apparentes.

Les sommets des lits d'hiver présentent un contact franc de type érosif. On y observe fréquemment de petites déformations pénécontemporaines à la mise en place, du type "structure en flammes". 


\section{Granulométrie}

La répartition granulométrique moyenne des varves échantillonnées à cinq niveaux différents à l'intérieur des séquences de SBG (sable 0,3 \%; silt 38,2\% et argile $61,5 \%$ ) et de NDN (sable $\approx 0,1 \%$; silt $33,4 \%$ et argile $66,5 \%$ ) peuvent être attribuées au faciès d'eau profonde, tel que défini par Veillette (1983b) à l'est du lac Témiscamingue (fig. 8). Dans les deux séquences varvaires, on observe la dominance des silts dans les lits d'été $(60,7 \%)$ et celle des argiles dans les lits d'hiver $(88,9 \%)$. On ne peut déceler d'évolution granulométrique en fonction du temps: la répartition granulométrique des varves (sable + silt; argile) est demeurée très constante de la base au sommet des séquences étudiées (fig. 9).

\section{Épaisseur}

Les varves de SBG ont une épaisseur variant entre 1,1 et $4,0 \mathrm{~cm}$. Leur épaisseur moyenne est de $2,1 \mathrm{~cm}$. Celle-ci se répartit presque également entre les lits d'été $(1,0 \mathrm{~cm}$ en moyenne) et les lits d'hiver $(1,1 \mathrm{~cm})$. Celles de NDN sont légèrement plus épaisses: $2,7 \mathrm{~cm}$ en moyenne. Cette différence est due à l'épaisseur plus grande des lits d'hiver $(1,7 \mathrm{~cm})$, les lits d'été montrant la même épaisseur moyenne qu'à SBG $(1,0 \mathrm{~cm})$. L'épaisseur maximale observée pour une varve est de $7,9 \mathrm{~cm}$ (fig. 6).

Afin de mieux visualiser l'évolution globale de l'épaisseur des varves, les moyennes des couplets ont été calculées

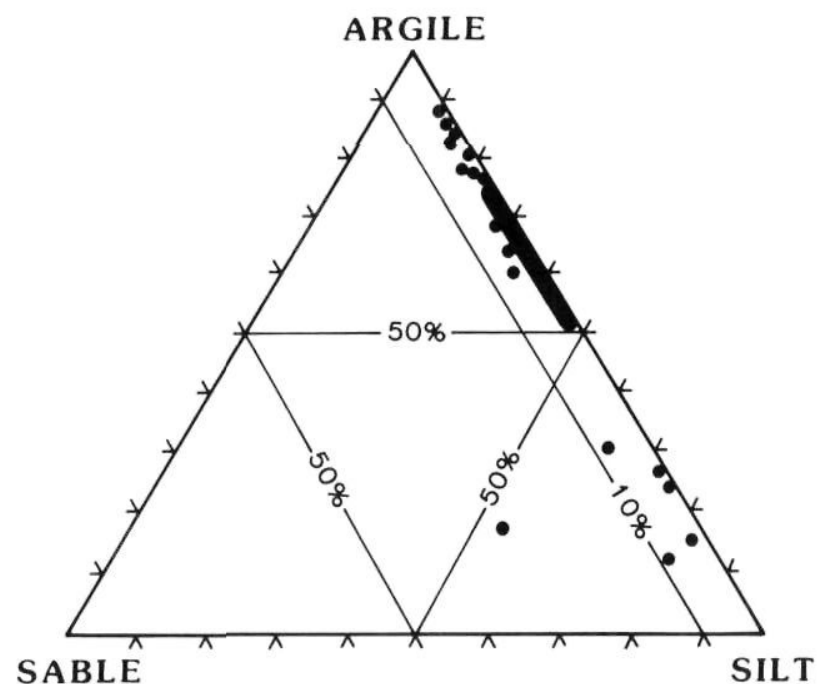

- Varves du faciès profond échantillonnées â l'est du lac Témiscamingue (Veillette, 1983b)

- Varves de St-Bruno-de-Guigues et de Notre-Dame-du-Nord

FIGURE 8. Répartition granulométrique des varves sur diagramme triangulaire; comparaison avec les données de Veillette (1983b).

Granulometric data of the varves on ternary diagram. Comparison with the analysis of Veillette (1983b).

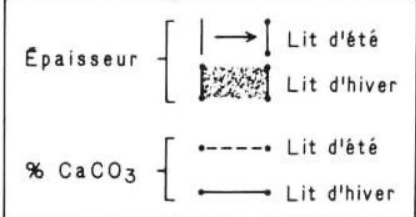

SEQUENCE VARVAIRE DE ST-BRUNO-DE-GUIGUES
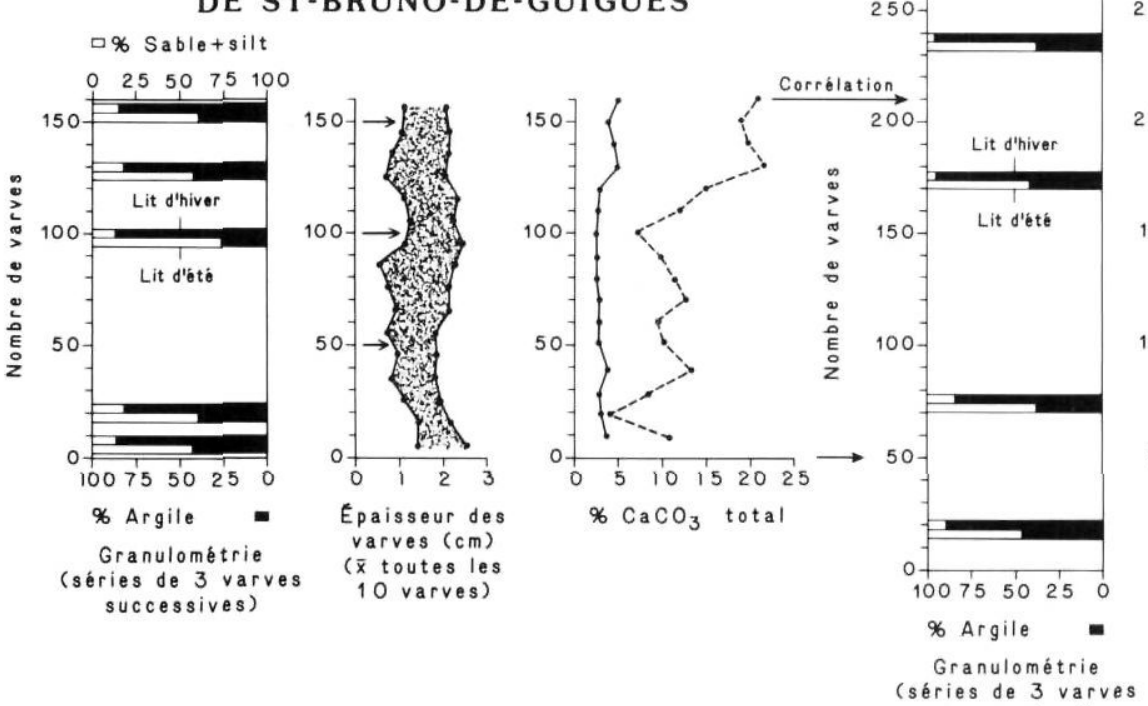

Sedimentological data of the varve sequences. Correlation: varve

FIGURE 9. Données sédimentologiques des séquences varvaires et corrélation: la varve 1 de Saint-Bruno-de-Guigues (SBG) correspond à la varve 51 de Notre-Dame-du-Nord (NDN). successives)

\section{SEQUENCE VARVAIRE DE NOTRE-DAME-DU-NORD}

$$
\begin{aligned}
& \square \% \text { Sabletsilt } \\
& 0 \quad 2550 \quad 75 \quad 100
\end{aligned}
$$
1 at SBG corresponds to varve 51 at NDN. 
pour des périodes de 10 ans (fig. 9). Les varves les plus épaisses de la coupe de SBG se situent à la base de la séquence: les lits d'été y sont plus importants. Par la suite, ces derniers diminuent en épaisseur jusqu'à la varve 90. On note cependant un épaississement global des varves à partir de la varve 60 , attribuable à l'augmentation de l'épaisseur des lits d'hiver.

À NDN, les varves les plus épaisses se situent également à la base de la coupe; les lits d'été forment la plus grande épaisseur du couplet. Cette tendance s'estompe rapidement, si bien qu'à partir de la varve 80 et jusqu'à la fin de la séquence étudiée, on observe le phénomène inverse, soit la prédominance du lit hivernal.

\section{Teneur totale en carbonate}

Dans les deux séquences varvaires, les teneurs en carbonates fluctuent (fig. 9). De façon générale, il existe une certaine corrélation entre les fluctuations présentes dans les lits d'été et ceux d'hiver (SBG: 0,46; NDN: 0,8): cependant ces derniers sont en moyenne 3,7 fois moins riches que les lits d'été.

Ces fluctuations s'inscrivent dans une tendance générale à la hausse des teneurs totales en carbonate. Les lits d'été de SBG passent d'une teneur de $11 \%$ à la base de la séquence à $21 \%$ au sommet, pendant que les lits d'hiver varient de 3,7 à $5,0 \%$. À la coupe de NDN, on remarque la même tendance: les lits silteux passent de $15,2 \%$ à $26 \%$ tandis que les lits argileux augmentent de 6,0 à 6,7\%.

\section{Composition minéralogique des argiles}

Les minéraux argileux des varves de SBG et de NDN sont les mêmes que ceux observés dans le till de SBG soit: feldspaths, dolomite, calcite, quartz, amphibole et phyllosillicates de type chlorite, micas et illite (fig. 10). Qualitativement cette composition ne varie pas dans le temps, comme l'ont démontré les diffractogrammes des varves SBG 10 et 150 ainsi que NDN 62 et 319.

\section{INTERPRÉTATION DES DONNÉES SÉDIMENTOLOGIQUES ET CORRÉLATION}

\section{Généralités}

L'observation des épaisseurs totales des varves et des lits qui les composent met en évidence l'existence de fluctuations dans les apports de sédiments.

À la base des coupes, les varves plus épaisses et la prédominance des lits grossiers (été) sur les lits fins (hiver) indiquent la proximité de la glace. Par la suite, la prédominance des lits fins indique le passage à des varves plus distales; il faut noter, cependant, que l'épaisseur des lits grossiers demeure relativement constante. II est probable que ce phénomène traduise un ralentissement local de la fonte (épaisseur constante des lits d'été), alors qu'elle se poursuivrait régionalement (augmentation des épaisseurs des lits d'hiver).

On associe l'augmentation des teneurs totales en carbonate des varves à un enrichissement de la glace en débris car-

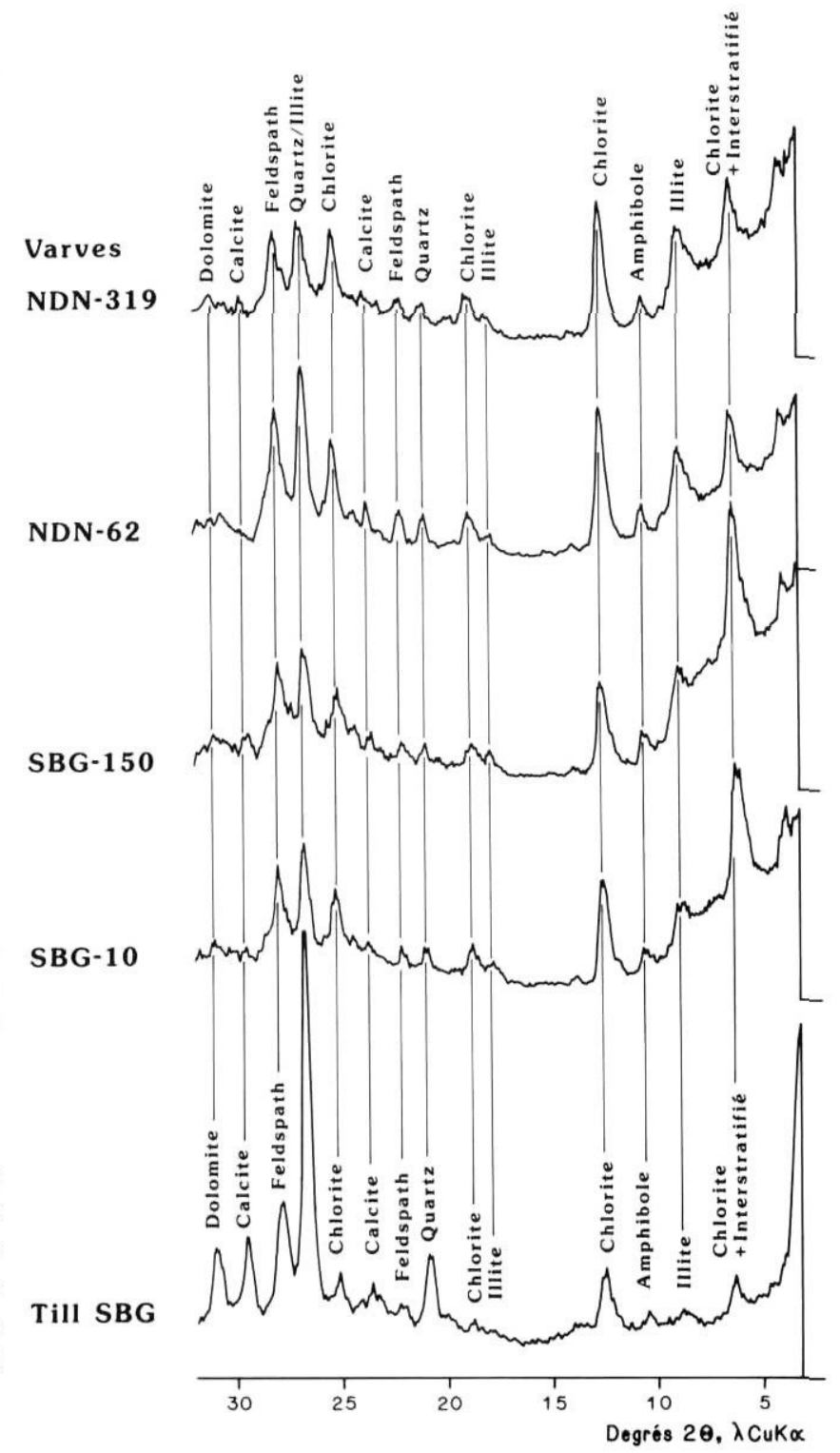

FIGURE 10. Diffractogrammes aux rayons $X$ de la fraction argileuse $(<2 \mu \mathrm{m})$ du till et de quelques varves.

$X$ ray diagrams of the clay fraction $(<2 \mu \mathrm{m})$ of the till and of a few varves.

bonatés attribuable à son confinement progressif sur le lambeau de roches paléozoïques, préservé dans la partie nord du graben du lac Témiscamingue. La présence tardive de ce lobe de glace a été supposée par Veillette (1982, 1983a et b). Cette augmentation des teneurs en carbonate ne peut s'expliquer par une variation granulométrique: aucune tendance n'est notée dans les séquences varvaires considérées. Cependant, la teneur relativement plus faible en carbonate des lits d'hiver (3,7 fois moins) par rapport aux lits d'été est probablement causée par une moindre quantité de silt $(11 \%$ en moyenne contre $61 \%$ pour les lits d'été, soit un rapport de 5,5 comparable à celui des carbonates). Rappelons que Dreimanis et Vagners (1971) ont pu évaluer que la taille terminale des minéraux carbonatés des roches de type paléozoïque 
se situait dans la classe granulométrique des silts $(0,004$ à $0,062 \mathrm{~mm}$ ).

L'analyse qualitative de la composition minéralogique des argiles des varves, par diffraction aux rayons $\mathrm{X}$, démontre que les apports de sédiments des lits estivaux et hivernaux proviennent d'une même source et que celle-ci s'apparente au till mis en place à SBG (fig. 10). Cette composition minéralogique polygénique reflète l'héritage pétrographique de la glace en raison de sa provenance NNE (roches précambriennes) auquel s'est ajoutée une composante locale (roches paléozoïques).

\section{Corrélation entre les deux séquences varvaires étudiées}

II existe bien entre les deux séquences étudiées des similitudes dans les tendances évolutives des valeurs moyennes de l'épaisseur des varves. Dans les deux cas, on note une diminution puis une légère augmentation des épaisseurs. Cette augmentation est principalement due à des apports hivernaux plus importants.

Selon Banerjee (1973, p. 16), ce sont les lits d'été qui montrent les meilleures possibilités de corrélation: "The latter means that silt layers have more "individuality", or a more distinctive thickness profile peculiar to each layer».

Les deux séquences ont donc été corrélées selon les variations d'épaisseur des lits d'été; on obtient la meilleure correspondance graphique et le coefficient de corrélation statistique le plus élevé $(0,56)$, lorsque la valeur moyenne des dix premières varves de Saint-Bruno-de-Guigues est corrélée avec celle de l'intervalle 50 à 60 de Notre-Dame-du-Nord (fig. 9). Si l'on compare par ailleurs les variations des teneurs en carbonates des lits estivaux, on remarque aussi une très bonne correspondance graphique et statistique entre les deux séquences: le coefficient de corrélation atteint 0,81 .

En considérant cette hypothèse de corrélation, on peut avancer certaines observations éclairant la paléogéographie:

(a) II existe au site de Notre-Dame-du-Nord (NDN), une certaine épaisseur de varves antérieures à la séquence varvaire de Saint-Bruno-de-Guigues (SBG): d'une part les 50 varves, tel que démontré précédemment par les corrélations; d'autre part 1,5 $\mathrm{m}$ de varves distales sous le niveau de l'eau. Compte tenu de l'épaisseur moyenne des 10 premières varves audessus du niveau de l'eau $(4,5 \mathrm{~cm})$, on peut estimer qu'environ 30 varves sont cachées.

C'est donc un total de l'ordre de 80 varves distales antérieures à celles de SBG qui sont présentes à NDN, (fig. 6).

Notons en outre que dans les deux coupes, on est en présence de varves proximales dont il est difficile de tenir compte dans cette reconstitution: à SBG, on a identifié environ 10 varves dans $0,50 \mathrm{~m}$; et à NDN il existe encore $1,5 \mathrm{~m}$ environ de matériel sablo-silteux stratifié (données de forage). II est donc possible qu'il existe un plus grand nombre de varves proximales à NDN. Toutes conditions étant égales, on peut donc penser que l'écart de 80 varves est minimal.

On estime donc à environ 80 ans la période de temps nécessaire au recul de la position de NDN à celle de SBG, situé $10 \mathrm{~km}$ plus au sud (fig. 2). On doit considérer ainsi l'existence d'une échancrure d'orientation NO-SE dans la masse de glace. Cette hypothèse concorde avec le modèle de déglaciation de Veillette (1982, 1983 a et b), modèle caractérisé par l'irrégularité de la marge glaciaire.

(b) On peut déduire le taux de retrait glaciaire en tenant compte des 80 années nécessaires au recul de la glace entre NDN et SBG et de la disposition des segments morainiques. Selon l'allure arquée de ces derniers, on suppose que la marge glaciaire devait se situer près du segment morainique de la pointe Millar (4 km au SSO de NDN; fig. 2) lorsque le site de SBG fut déglacé. On estime ainsi le taux de retrait moyen (maximal) à $50 \mathrm{~m}$ par année. Ce taux est nettement inférieur à celui obtenu par Antevs (1925; $138 \mathrm{~m} / \mathrm{an}$ ) pour le retrait de la marge glaciaire du bassin du Témiscamingue; on aurait donc un ralentissement important de la fonte dans ce secteur.

Veillette (1983a) considère que la présence d'un lobe de glace dans le graben du lac Témiscamingue est synchrone avec la construction de la Moraine de Roulier, à $30 \mathrm{~km}$ au nord. La formation de cette moraine indiquerait une stagnation de la marge glaciaire et confirmerait l'hypothèse d'un ralentissement de la fonte de l'inlandsis dans le secteur étudié.

(c) L'étude des variations de l'épaisseur totale des varves des deux séquences analysées ne traduit pas l'évolution d'un lobe de glace vers un culot qui aurait fondu sur place dans l'auge du lac Témiscamingue, comme l'a cru Veillette (1983 a). En effet, on n'observe aucune augmentation importante de l'épaisseur des varves. Si cette évolution a eu lieu, elle s'est effectuée postérieurement à l'intervalle de temps enregistré dans la plus longue des séquences varvaires étudiées, celle de NDN (333 varves).

Corrélation des séquences étudiées avec les séries du Témiscamingue d'Antevs (1925)

Afin de corréler les séquences varvaires étudiées avec celles d'Antevs (1925), une courbe montrant la variation d'épaisseur des varves dans le temps a été construite (fig. 11).

Les corrélations empiriques sont effectuées en faisant glisser les courbes les unes sur les autres jusqu'à l'obtention de la meilleure correspondance possible. Notons que les courbes d'Antevs ont été construites en faisant la moyenne des épaisseurs d'une même varve à différents lieux. Dans les cas étudiés, vu l'approximation du nombre de varves impliquées dans les quelques horizons déformés, la corrélation des plus longues séquences non perturbées de chacune des deux coupes a été tentée. Selon la corrélation établie précédemment, la varve 50 de SBG correspondrait à la varve 100 de la coupe de NDN. En faisant la moyenne des épaisseurs des varves correspondantes, la courbe moyenne des deux séquences étudiées a été établie.

D'après les données d'Antevs (1925), la région de NDN devait être déglacée vers "l'an 350 » de sa chronologie. Compte tenu du fait que la courbe moyenne proposée ici commence à la varve 100 de la coupe de NDN, et en tenant compte qu'il existe environ 30 varves sous le niveau de l'eau à ce site, 

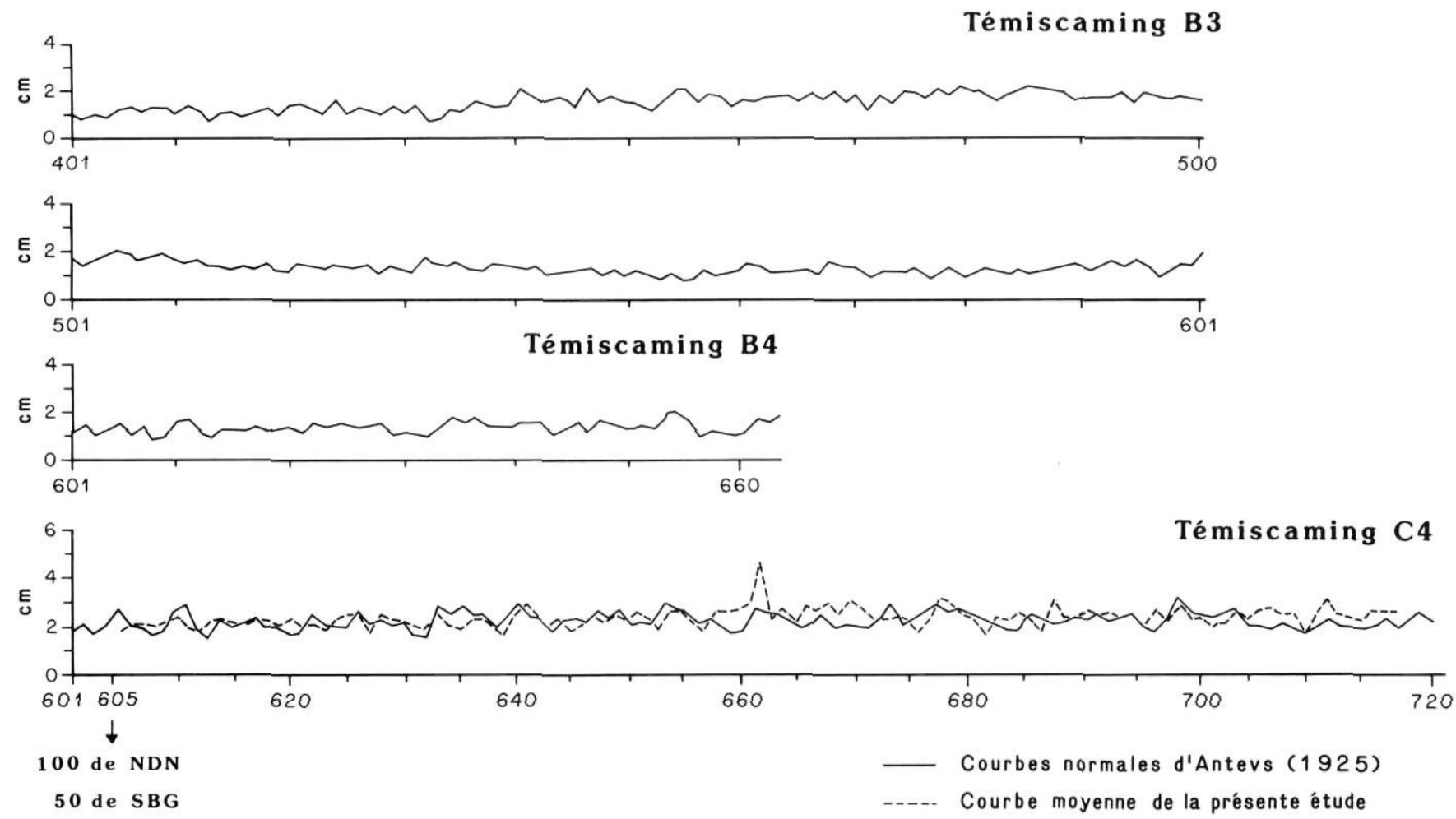

FIGURE 11. Corrélation entre la courbe d'épaisseur moyenne des varves étudiées et celles d'Antevs (1925).

Correlation between the mean thickness curve of the studied varves with those of Antevs (1925).

on doit conclure que cette courbe correspond à l'intervalle compris entre les varves 480 et 593 de la chronologie d'Antevs. La courbe, construite à partir des sites les plus proches de nos coupes, et qui couvre cet intervalle de temps, a été nommée par Antevs (1925) Temiskaming B3 (fig. 11). Elle résulte des données d'épaisseur de varves de six coupes près de Laverlochère ( $25 \mathrm{~km}$ au SE des coupes étudiées). II ne paraît pas possible de corréler la nouvelle séquence avec les données de Temiskaming B3. En fait, les variations d'épaisseur de cette séquence correspondent beaucoup plus à la courbe Temiskaming B4, provenant également de Laverlochère mais comprenant les varves 601 à 664 , et à la courbe Temiskaming $\mathrm{C} 4$, identifiée près d'Haileybury en Ontario (20 km au SO des coupes étudiées), et commençant à la varve 605 .

La corrélation de notre courbe avec des varves plus récentes de la chronologie d'Antevs confirmerait donc la présence tardive de glace dans le secteur d'étude: en effet, si l'on trace la position de la marge glaciaire à +500 de la chronologie d'Antevs, celle-ci doit passer entre les sites étudiés (NDN: $1^{\text {re }}$ varve retracée $=475 ;$ SBG: $1^{\text {re }}$ varve $=545$ ), et un peu au sud des sites 82 et 83 où Antevs a respectivement identifié les varves 594 et 592, directement sur le till (fig. 12).

Cette configuration de la marge glaciaire concorde avec les écoulements glaciaires tardifs, la disposition des sédiments de contact glaciaire de la région et avec l'augmentation des teneurs en carbonates des varves.

\section{LA MORAINE DE LAVERLOCHĖRE}

\section{MÉTHODOLOGIE}

L'importance du transport glaciaire provoqué par la présence tardive du lobe a été évalué par l'analyse du contenu pétrographique des divers segments de la Moraine de Laverlochère. Au total, 14 gravières ont été échantillonnées (fig. 13). À chaque site, on a effectué un comptage pétrographique sur les galets (Axe $A=30-80 \mathrm{~mm}$ ). Ceux-ci ont été recueillis en place et à la base des coupes observées, généralement vers le centre des accumulations. On a distingué quatre catégories de roches: (1) les roches volcaniques, les granites et les gneiss archéens; (2) les conglomérats et les argilites de la Formation de Gowganda; (3) les quartzites de la Formation de Lorrain; (4) les roches carbonatées provenant des lambeaux de roches paléozoïques. La localisation de ces lithologies apparaît à la figure 13: notons cependant que la précision de la cartographie dans ce secteur est limitée par la présence des sédiments glaciolacustres.

Par ailleurs, l'étude des sédiments dont le diamètre est inférieur à $8 \mathrm{~mm}$ avait aussi pour objectif de déterminer leur teneur en débris de roches carbonatées. Après tamisage à sec, la quantité absolue de carbonates fut déterminée pour les fractions granulométriques suivantes: $2-8 / 1-2 / 0,5-1 /$ $0,25-0,5 / 0,125-0,25 / 0,063-0,125 /<0,063$ mm. Pour la classe comprise entre 2 et $8 \mathrm{~mm}$, on a procédé par identification visuelle d'une population de 100 débris. Pour les autres frac- 
tions, on a déterminé la teneur totale en carbonate à l'aide d'un calcimètre de type Bernard.

Afin de mieux comprendre le mode de mise en place de la Moraine de Laverlochère des mesures de direction des

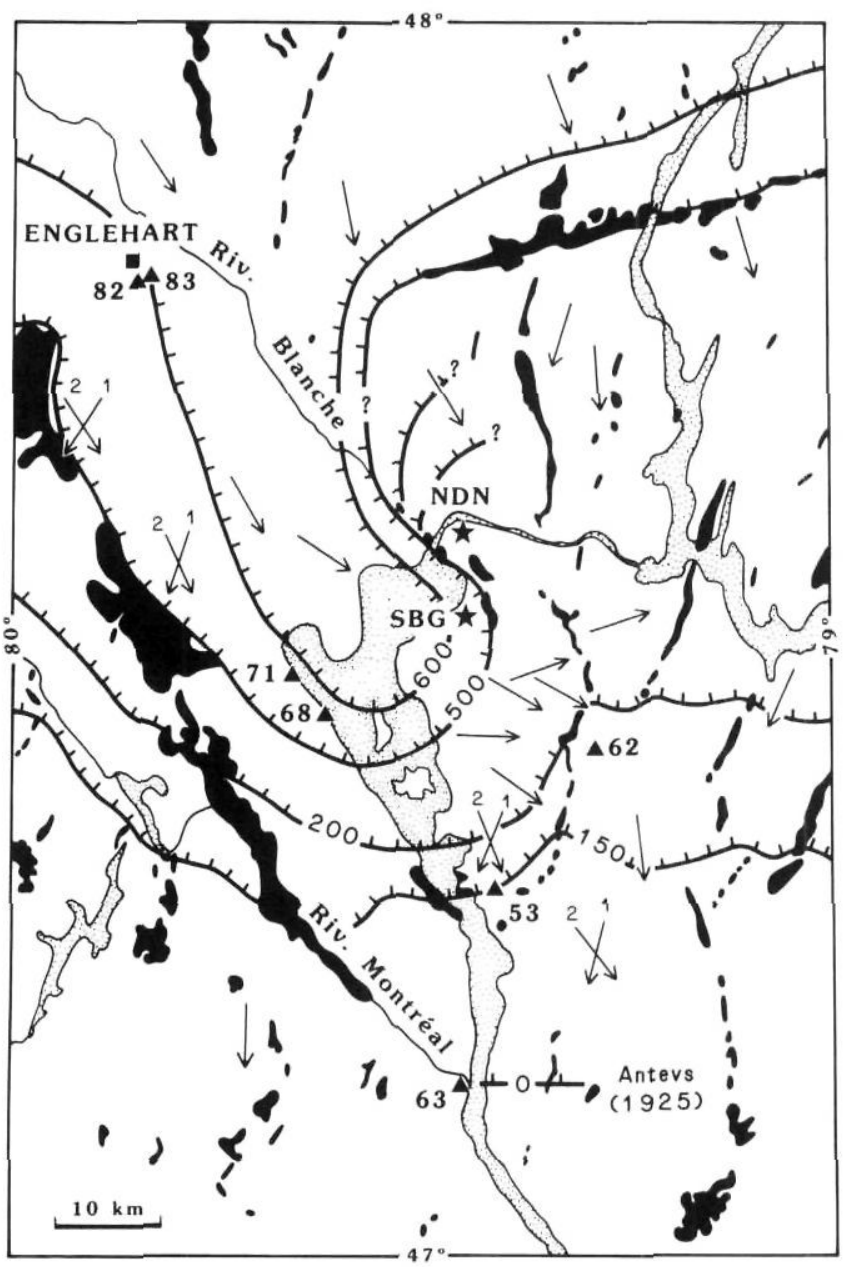

$\star$ NDN: Notre-Dame-du-Nord, Varve 475 observée

\ SBG: Saint-Bruno-de-Guigues, Varve 545 sur till

- Site d'Antevs (1925)

63: Varve 1 sur till

53: Varve 142 sur till

62: Varve 185 sur substratum rocheux

68,71: Varve 580 observée

83: Varve 592 sur till

82: Varve 594 sur till

$\longrightarrow$ Écoulements glaciaires déterminés par des marques d'érosion $(1,2$ : chronologie)

\footnotetext{
Sédiments fluvioglaciaires

500 Position approximative de la marge glaciaire à +500 de la chronologie d'Antevs

$\sim \operatorname{LaC}$
}

FIGURE 12. Reconstitution paléogéographique: positions de la marge glaciaire lors du retrait de l'inlandsis. Écoulements glaciaires et sédiments fluvioglaciaires de contact d'après Veillette (1986a, b, c).

Paleogeographic framework: ice-frontal positions during the late ice sheet retreat. Ice-flow directions and ice-contact deposits from Veillette $(1986 a, b, c)$ "paléocourants" ont également été effectuées: en fait on considère les paléopentes sédimentaires comme étant statistiquement parallèles aux directions du transport des matériaux.

\section{RÉSULTATS}

La figure 13 présente l'ensemble des résultats sédimentologiques obtenus à partir des sédiments de la Moraine de Laverlochère.

\section{Composition lithologique}

Du point de vue lithologique, le fait majeur consiste en la prédominance des roches archéennes (exception faite du site $\left.n^{\circ} 14\right)$, même dans le secteur des roches protérozoïques (formations de Lorrain et de Gowganda). Le plus fort pourcentage cumulatif de ces dernières $(30 \%)$ est observé au site le plus au sud (site $n^{\circ} 12$ ); ailleurs on en compte généralement moins de $10 \%$, sauf au site $\mathrm{n}^{\circ} 1(22 \%)$.

Les galets carbonatés se concentrent dans deux zones: dans le secteur de SBG et au S et SE de Ville-Marie, soit respectivement dans les segments morainiques situés immédiatement à l'E et au SE du lambeau de roches paléozoïques. La concentration maximale $(47 \%)$ est observée au site $n^{\circ} 14$. Notons qu'un échantillon de till provenant de la base des varves de SBG, donc reposant sur le substratum paléozoïque (fig. 7 et fig. 13 , site $n^{\circ} 15$ ), contient $14 \%$ de galets carbonatés (de 0,8 à $5 \mathrm{~cm}$ ), alors qu'un diamicton glaciolacustre (till en coulée ou coulée boueuse), qui lui succède, en contient $28 \%$; sa teneur en roches protérozoïques demeure stable à environ $25 \%$.

Tout comme pour les galets, les pourcentages de carbonates obtenus par calcimétrie sont plus élevés dans les segments morainiques situés à proximité, et à l'ESE, du lambeau de roches paléozoïques (fig. 14; site $\mathrm{n}^{\circ \mathrm{s}} 2$ et 14). Au site $\mathrm{n}^{\circ} 2$, trois échantillons prélevés à $-15 \mathrm{~m},-9 \mathrm{~m}$ et $-7 \mathrm{~m}$ du sommet de la moraine ont été analysés. Ces échantillons montrent une distribution similaire des carbonates dans les différentes classes granulométriques. Ceux-ci, comme ceux des sites $n^{\circ} 1$ et 14 , présentent une bimodalité dans la distribution des carbonates de la matrice: un mode dans les débris grossiers (sable grossier), et un mode dans les débris fins (sable très fin et lutites). Les pourcentages de carbonates obtenus par identification visuelle dans les fractions granulométriques supérieures à $2,0 \mathrm{~mm}$ semblent toutefois indiquer que le mode grossier domine dans la classe des rudites.

En s'éloignant de la source des roches carbonatées, la fraction carbonatée du mode grossier tend à diminuer (sites nos 11 et 12). Dans les segments les plus éloignés vers l'E, à l'exception du site $n^{\circ} 7$, on trouve très peu de carbonates, et ceux-ci se concentrent essentiellement dans les lutites.

\section{Direction des paléocourants}

La figure 13 montre la direction des "paléocourants" mesurés dans les divers segments morainiques. On y note une grande variabilité entre les sites: aux sites nos 2 et 14 les pe léocourants se dirigent respectivement vers le $\mathrm{N}$ et le $\mathrm{NE}$; au site $n^{\circ} 4$ vers l'E; aux sites nos $6,10,11$ et 13 vers le $S$, 


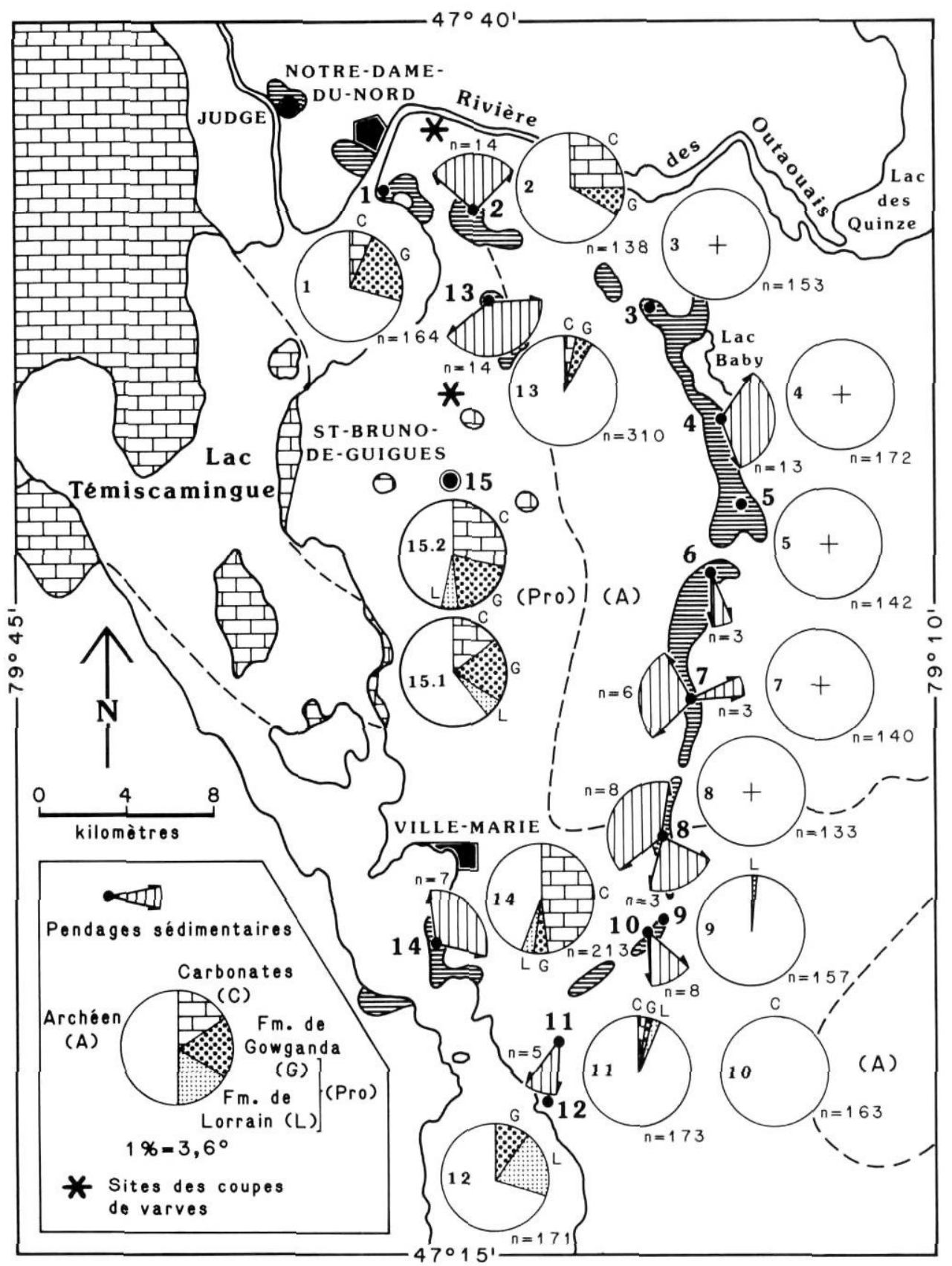

FIGURE 13. Étude sédimentologique de la Moraine de Laverlochère: pétrographie des galets (30 à $80 \mathrm{~mm}$ ) et paléocourants. Pour la géologie du substratum voir la figure 1. Traits horizontaux: Moraine de Laverlochère; Pro: Protérozoïque.

Sedimentological study of the Laverlochère Moraine: lithology of the pebbles $(30-80 \mathrm{~mm})$ and paleocurrents. For the bedrock geology see Figure 1. Horizontal dashed line: the Laverlochère Moraine; Pro: Proterozoic.

tandis qu'aux sites $n^{\circ} 7$ et 8 on note des directions opposées soit respectivement vers l'O ou l'E et vers le NO ou SE.

\section{DISCUSSION}

La prédominance des roches archéennes sur les autres groupes de roches reflète, comme la teneur en minéraux argileux des varves, I'héritage lithologique de la glace qui provenait du secteur NNE. En tenant compte de cet héritage pétrographique "normal" et de la proximité du contact avec les roches archéennes, la teneur en galets protérozoïques des sites nos 1,2 et 13 , comme celui du till et du diamicton cle SBG, est relativement beaucoup plus élevé que celui des sites nos $9,10,11,12$ et 14 . Cette différence peut s'expliquer par un transport glaciaire tardif, généralement d'O en $\mathrm{E}$, dans ce secteur. Cette hypothèse est confirmée par la présence de galets carbonatés aux mêmes sites nos 1,2 et 13. Le fort pourcentage de roches carbonatées au site $n^{\circ} 14$, comparativement à ceux mesurés à ces derniers sites, pourrait démontrer qu'il existe d'autres affleurements de roches paléozoïques, non cartographiés, à proximité de ce segment morainique. Et la direction des paléocourants indiquerait que cet affleurement est probablement localisé dans l'auge du lac Témiscamingue. Toutefois, le transport vers le SE sur une plus longue distance pourrait également avoir été favorisé par la dépression.

Puisque la présence de galets de carbonates n'est observée que dans les segments morainiques situés à proximité du 


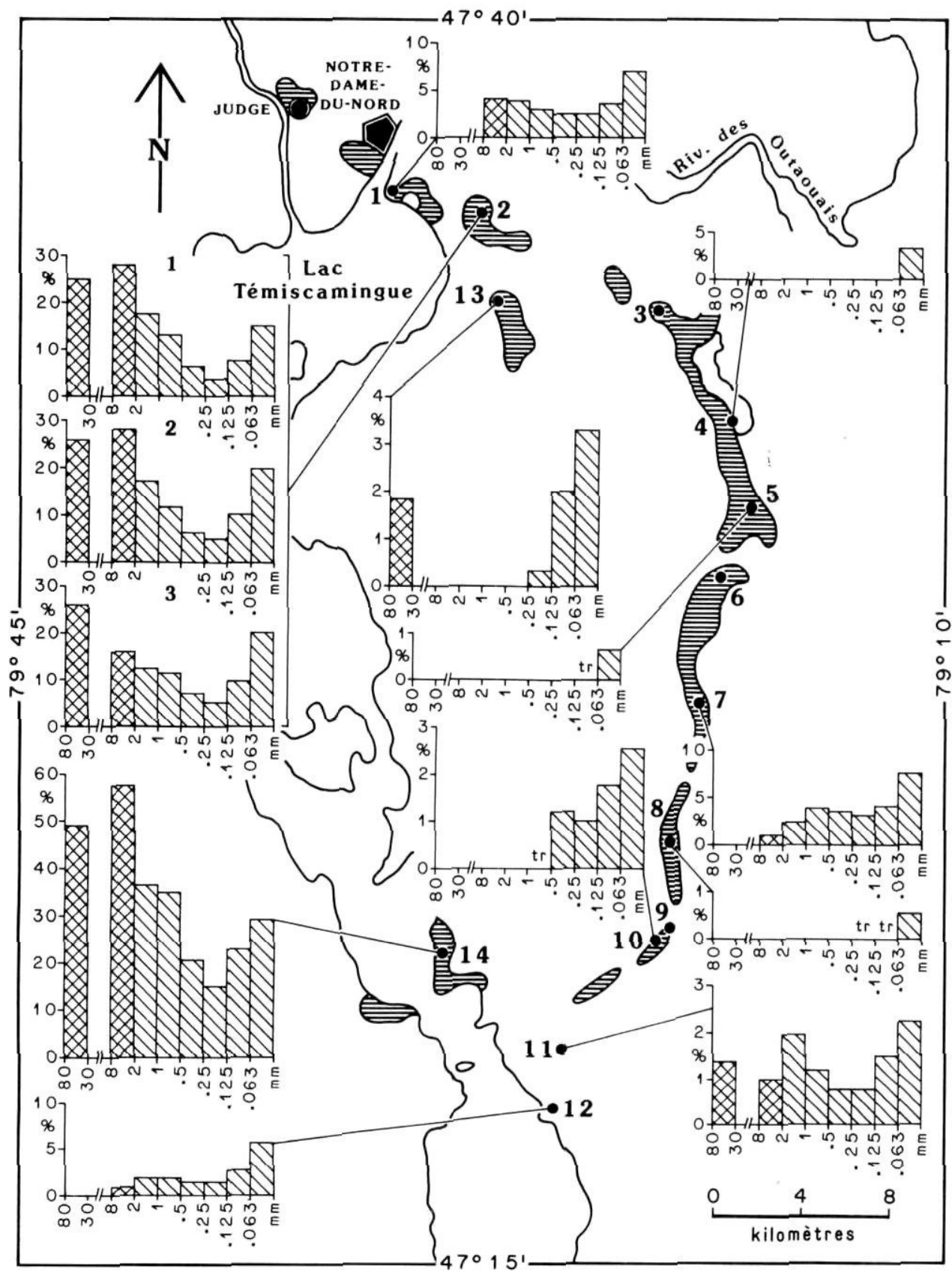

FIGURE 14. Teneur totale en carbonates des diverses fractions granulométriques des dépôts de la Moraine de Laverlochère. Zone hachurée: comptage; zone lignée simple: calcimétrie.

Total carbonate content of various grain-sizes from the Laverlochère Moraine. Hatched zone: count; oblique ligne zone: calcimetry.

lambeau de roches paléozoïques, on doit conclure que le transport des débris de cette taille fut faible: la décroissance rapide du pourcentage de galets carbonatés du site $n^{\circ} 14$ au site $n^{\circ} 11$, dans le sens de l'écoulement glaciaire tardif observé pour ce secteur, soit vers le SE, le confirme.

La bimodalité des teneurs en carbonate des fractions plus fines $(<2 \mathrm{~mm})$ dans les sites adjacents au lambeau paléozoïque indique également un certain transport. Dans les sites plus orientaux ( $n^{\text {os }} 4,5,7,8$ et 10), les teneurs des fractions fines sont du même ordre que celles mesurées par J. Veillette (comm. pers.) dans la matrice des tills du Témiscamingue, composés de roches volcaniques: elles ne peuvent, à priori, être reliées à un apport de carbonates du lambeau de roches paléozoïques. On peut donc conclure que le transport des débris fins se fit sur une courte distance, comme pour les galets.

Au site $n^{\circ} 7$, on explique la richesse relative en carbonates par le lessivage des varves qui surmontent les sédiments morainiques et la précipitation secondaire de la calcite dans les sables et graviers: en effet, des amas de graviers cimentés par de la calcite secondaire ont été observés dans cette gravière.

Aux sites nos $2,4,6,9,11,13$ et 14 , les directions des paléocourants observées tendent à confirmer la présence de la glace du côté ouest, soit à l'intérieur de la superficie délimitée par la moraine qui est arquée. Celles des sites $n^{\text {os }} 7$ et 8 indiqueraient la présence de la glace de part et d'autre du corps sédimentaire. La Moraine de Laverlochère pourrait ainsi 
avoir connu un mode de mise en place composite, c'est-àdire tantôt en position frontale, et, pour d'autres secteurs, plutôt de type esker. Ainsi, on suppose un mode de mise en place sous forme d'esker pour les segments orientaux de la moraine à partir des indices suivants: 1) leur disposition spatiale est subparallèle à l'orientation des autres complexes fluvioglaciaires de type esker, situés à l'E du lac Témiscamingue (Veillette, 1983b; fig. 15); 2) La direction des paléocourants, du moins dans les segments où se trouvent les sites nos 7 et 8 , indiquerait la présence de la glace de part et d'autre des accumulations; 3 ) la teneur en carbonates des sédiments démontre que leur mise en place est antérieure au confinement de la glace sur le lambeau de roches paléozoïques et ne diffère pas sensiblement des teneurs en carbonates des matrices des tills régionaux renfermant seulement des roches précambriennes; 4) enfin, dans la partie sud de la zone étudiée, les marges glaciaires établies à partir des varves mesurées par Antevs (1925) seraient plutôt perpendiculaires et non parallèles à ces segments fluvioglaciaires. II en découle ainsi un certain diachronisme dans la mise en place de ces accumulations fluvioglaciaires (fig. 12). En fait, seuls les segments de Judge et de Notre-Dame-du-Nord et ceux des sites nos 1 , 2,13 et 14 (fig. 13) représenteraient deux positions frontales qui ne seraient pas synchrones (fig. 12).

L'analyse de la direction des paléocourants et du contenu pétrographique des divers segments morainiques indique qu'il y a eu écoulement et transport vers l'E et le SE, tel que proposé par Veillette (1983a et 1986) dans son modèle de déglaciation fondé principalement sur les marques d'érosion. Ce transport est cependant faible et se limite au secteur situé immédiatement à l'est et au SE du lambeau de roches paléozoïques. Les segments morainiques $n^{\text {os }} 1,2$ et 13 , au NE du lac Témiscamingue et ceux de Notre-Dame-du-Nord et de Judge sont contemporains au lobe de glace tardif centré sur la partie nord du graben du lac Témiscamingue (fig. 12).

\section{CONCLUSION}

Les corrélations varvaires établies (entre SBG et NDN; entre SBG + NDN et Temiskaming B4 et C4 d'Antevs, 1925) s'ajoutent aux données d'ordre géomorphologique et sédimentologique de la Moraine de Laverlochère pour démontrer l'irrégularité de la marge glaciaire lors de son retrait. Un ralentissement marquée du taux de retrait glaciaire $(50 \mathrm{~m} / \mathrm{an}$ contre $138 \mathrm{~m}$ selon Antevs, 1925) dans la partie nord du graben du lac Témiscamingue expliquerait la présence tardive d'un lobe de glace dans ce secteur comme l'ont supposé Barlow (1899) et Veillette (1982, 1983 a et b). Ce lobe de glace a subsisté durant plus de 300 ans, mais n'a entraîné qu'un faible transport vers l'E et le SE.

La Moraine de Laverlochère, telle que définie par Veillette (1983 a et b), ne délimite pas la bordure du lobe de glace tardif ayant occupé le graben du Témiscamingue. Elle est constituée dans sa partie orientale d'une série d'accumulations fluvioglaciaires diachroniques de type esker tel que proposé par Vincent (1971): l'appellation de "Moraine de Laverlochère " devrait donc être modifiée. Une meilleure connaissance de la répartition des dépôts fluvioglaciaires probablement enfouis sous les varves du bassin, et une étude systématique des séquences de varves elles-mêmes, apporteraient sans doute plus de précisions sur le mode de retrait glaciaire wisconsinien dans la région.

\section{REMERCIEMENTS}

Nous tenons à remercier: pour l'assistance sur le terrain M. P. Blais (1983); pour les traitements aux rayons $X$ des argiles, M. M. Preda; pour la dactylographie du manuscrit, $\mathrm{M}^{\mathrm{me}}$ Manon Brassard; pour la réalisation des figures, $\mathrm{M}^{\mathrm{me}}$ Michelle Laithier. Nous exprimons également notre gratitude à M. J. Veillette pour l'organisation matérielle et ses encouragements. Les travaux ont été subventionnés par la Commission géologique du Canada et par une bourse du C.R.S.N.G. Les suggestions de MM. Jean Veillette et Serge Occhietti, lecteurs de la revue, ont été appréciées.

\section{RÉFÉRENCES}

Antevs, E., 1925. Retreat of the last ice sheet in eastern Canada. Geological Survey of Canada, Memoir 146, 142 p.

1953. Geochronology of the Deglacial and Neothermal ages. Journal of Geology, 61: 195-230.

Banerjee, I., 1973. Sedimentology of Pleistocene glacial varves in Ontario, Canada. Geological Survey of Canada, Bulletin 226, $60 \mathrm{p}$.

Barlow, A. E., 1899. Geology and natural resources of the area included by Nipissing and Temjscaming map-sheets comprising portions of the District of Nipissing, Ontario, and the County of Pontiac, Quebec. Report of the Geological Survey of Canada, 672: 18207.

Boissonneau, A. N., 1968. Glacial history of northeastern Ontario II. The Temiskaming-Algoma area. Canadian Journal of Earth Sciences, 5: 97-109.

Bostock, H. S., 1972. Subdivisions physiographiques du Canada, p. 12-34. In Géologie et ressources minérales du Canada. Commission géologique du Canada.

Donaldson, J. A. et Munro, I., 1982. Precambrian geology of the Cobalt area, northern Ontario. I.A.S. Guide d'excursion 16b, 72 p.

Dreimanis, A. et Vagners, U. J., 1971. The effect of lithology upon texture of till, p. 66-81. In E. Yatsu et A. Falconer, édit., Research methods in Pleistocene geomorphology.

Hughes, O. L., 1955. Surfacial geology of Smooth Rock and Iroquois Falls map areas, Cochrane District, Ontario. Ph.D. Thesis, University of Kansas, Lawrence, $190 \mathrm{p}$.

1965. Surficial geology of part of the Cochrane District, Ontario, Canada, p. 535-565. In H.E. Wright Jr. et D.E. Frey, édit., International Studies on the Quaternary, Geological Society of America Special Paper 84.

Kay, G. M., 1942. Ottawa-Bonnechere graben and the Lake Ontario homocline. Geological Society of America Bulletin, 53: 585.

Kumarapelli, P. S. et Saull, V. A., 1966. The St-Lawrence valley system: a north american equivalent of the east african rift valley system. Canadian Journal of Earth Sciences, 3: 639-658.

Lovell, H. L. et Caine, T.W., 1970. Lake Temiskaming rift valley Ontario. Department of Mines, MP 39, $16 \mathrm{p}$. 
Lunqvist, J., 1985. The 1984 symposium on clay-varve chronology in Stockholm. Boreas, 14: 97-99.

Nenniger et Chênevert, 1960. Rapport sur l'étude de la rivière des Quinze. 3 vol. Cie d'ingénieurs-conseils de Montréal, Montréal.

McGlynn, J. C., 1972. Géologie du Bouclier canadien, p. 50-165. In Géologie et ressources minérales du Canada, partie A. Commission géologique du Canada.

Mériaux, S., 1954. Contribution à l'étude de l'analyse granulométrique. Thèse de doctorat, Université de Paris, $118 \mathrm{p}$.

Prest, V. K., 1970. Quaternary geology of Canada, p. 676-784. In R. J. W. Douglas, édit., Geology and economic minerals of Canada, $5^{e}$ éd. Geological Survey of Canada, Economic Geology, Report 1.

Terasmae, J., 1963. Notes on palynological studies of varved clays. Journal of Sedimentary Petrology, 33: 314-319.

Veillette, J. J., 1982. Ice-flow patterns, Lake Temiscamingue area, Quebec. Commission géologique du Canada, dossier public 841, carte et commentaires.

1983a. Les polis glaciaires au Témiscamingue, une chronologie relative. Recherches en cours, Partie $A$, Commission géologique du Canada, Étude 83-1A: 187-196. 1983b. Déglaciation de la vallée supérieure de l'Outaouais, le lac Barlow et le sud du lac Ojibway, Québec. Géographie physique et Quaternaire, 37: 67-84.

1986a. Former southwesterly ice flows in the AbitibiTémiskaming region: implications for the configuration of the Late Wisconsinan ice sheet. Canadian Journal of Earth Sciences, 23: 1724-1741.

1986b. Géologie des formations en surface, New Liskeard, Ontario-Québec Commission géologique du Canada. Carte 1639A, échelle 1/100 000 .

1986c. Géologie des formations en surface, Haileybury, Ontario-Québec. Commission géologique du Canada, Carte 1642A, échelle $1 / 100000$.

Vincent, J.-S., 1971. Le Quaternaire des cantons de Guigues. Baby, Duhamel et Laverlochère, comté de Témiscamingue, Québec. Thèse de maîtrise, Université d'Ottawa. $146 \mathrm{p}$.

Vincent, J.-S. et Hardy, L., 1977. L'évolution et l'extension des lacs glaciaires Barlow et Ojibway en territoire québécois. Géographie physique et Quaternaire, 31: 357-372.

Wilson, M. E., 1918. Temiskaming county, Quebec. Geological Survey of Canada, memoir 103,197 p. 